\title{
Alternative Defaultable Term Structure Models
}

\author{
Nicola Bruti-Liberati $\dagger^{1}$, Christina Nikitopoulos-Sklibosios ${ }^{2}$, \\ Eckhard Platen $^{3}$ and Erik Schlögl ${ }^{2}$
}

\author{
January 16, 2009
}

\begin{abstract}
The objective of this paper is to consider defaultable term structure models in a general setting beyond standard riskneutral models. Using as numeraire the growth optimal portfolio, defaultable interest rate derivatives are priced under the real-world probability measure. Therefore, the existence of an equivalent risk-neutral probability measure is not required. In particular, the real-world dynamics of the instantaneous defaultable forward rates under a jump-diffusion extension of a HJM type framework are derived. Thus, by establishing a modelling framework fully under the real-world probability measure, the challenge of reconciling real-world and risk-neutral probabilities of default is deliberately avoided, which provides significant extra modelling freedom. In addition, for certain volatility specifications, finite dimensional Markovian defaultable term structure models are derived. The paper also demonstrates an alternative defaultable term structure model. It provides tractable expressions for the prices of defaultable derivatives under the assumption of independence between the discounted growth optimal portfolio and the default-adjusted short rate. These expressions are then used in a more general model as control variates for Monte Carlo simulations of credit derivatives.
\end{abstract}

Mathematics Subject Classification: primary 60H10; secondary 65C05. JEL Classification: G10, G13

Key words and phrases: defaultable forward rates, jump-diffusion processes, growth optimal portfolio, real-world pricing.

\section{Introduction}

This paper considers defaultable term structure models under the real-world probability measure. When modelling credit risk, the choice of an appropriate equivalent risk-neutral pricing measure has never been a straightforward task. Realistic

\footnotetext{
${ }^{1}$ In memory of our beloved colleague and friend.

${ }^{2}$ University of Technology Sydney, School of Finance \& Economics

${ }^{3}$ University of Technology Sydney, School of Finance \& Economics and Department of Mathematical Sciences
} 
market models, see Heath and Platen (2002a) and Platen (2002), may not even admit an equivalent risk-neutral probability measure. We argue in this paper that the real-world pricing approach provides a significant advantage over the traditional risk-neutral pricing framework and generalises the existing risk-neutral approach for pricing derivatives subject to default.

In a market driven by continuous and discrete trading uncertainty, we develop jump-diffusion interest rate term structure models by extending the classical Heath, Jarrow, and Morton (1992) (HJM) framework. As a natural application of jump-diffusion models, credit events including defaults are modelled as jumps. The paper derives the real-world HJM type dynamics of the instantaneous defaultable forward rates. The novelty of this result is that we obtain analogous dynamics of the defaultable forward rates, as for instance derived by Schönbucher (1998), yet they are described under the real-world probability measure and avoid the restrictive assumption on the existence of an equivalent risk-neutral probability measure. Since we do not rely on a change of the probability measure, the jump intensities we deal with are real-world intensities.

The proposed approach yields a consistent framework that has the power to connect real-world default probabilities and observed credit spreads under the real-world probability measure. This unifies the pricing and hedging of derivative instruments, traditionally performed under some putative risk-neutral probability measure, with the tasks of risk measurement such as VaR and portfolio optimization. The latter require in any case the use of the real-world probability measure. One can say that risk-neutral pricing is a kind of relative pricing. As soon as some market participant places some derivative price in the market, others can use it to calibrate their risk-neutral model and form consistent prices for further derivatives. This does not prevent a development where over long periods of time such prices can be way out from what may be realistically sustainable, as observed prior to the recent subprime crisis. Real-world pricing however, is a form of absolute pricing. Taking historical data and economic arguments into account one does not rely on the presence of some credit derivatives in the market for calibrating the model. This could have potentially avoided the situation where an entire industry failed to value correctly the risk in subprime credit derivatives. Real-world pricing is nothing but an investment decision where the investor values a claim with respect to his or her best performing portfolio, the growth optimal portfolio (GOP), without the potential distortions by using an artificial risk-neutral measure.

The fundamental principle of real-world pricing is that by taking as numeraire the GOP, pricing can be performed under the real-world or historical probability measure, see Platen and Heath (2006). More specifically, the value of a derivative is expressed in terms of a real-world conditional expectation. This provides the considerable advantage that the existence of an equivalent risk-neutral probability measure is not required. Consequently, a richer modelling world results than is available under the classical risk-neutral approach. As shown in Platen and 
Heath (2006), a diversified world stock index can be used as a proxy of the GOP. A particular form for the stochastic differential equation (SDE) of the GOP for a market driven by jump diffusions emerges. The generalised volatilities and the jump coefficients of the GOP characterize the market prices of diffusion and jump risk, respectively. The dynamics of the GOP are determined by these market prices of risk, as well as the risk-free short rate. In the literature, the market price of jump risk is usually assumed to be zero. The proposed approach allows to relax this assumption.

A class of alternative defaultable term structure models, which do not admit an equivalent risk-neutral probability measure, will be demonstrated. These general jump- diffusion models do not normally have closed form solutions, therefore Monte-Carlo simulation is the typical numerical approach to handle these models. However, standard Monte-Carlo simulation by using an Euler scheme or similar standard schemes may not be adequate. The proposed model requires the simulation of strictly positive affine processes. This type of processes typically encounters simulation problems when the process comes near zero under standard simulation schemes. This can be resolved by using an exact simulation scheme, see for instance Broadie and Kaya (2006). Furthermore, the efficiency of the Monte-Carlo simulation can be significantly improved by using variance reduction methods, see Kloeden and Platen (1999) and Heath and Platen (2002b).

In this paper, we demonstrate how a class of tractable defaultable term structure models can be obtained. More specifically, by assuming independence between the discounted GOP and the default-adjusted short rate, we first obtain closed form expressions for the prices of defaultable bonds and potentially other derivatives. Then in the more general case, we use an exact simulation scheme, in the spirit of Platen and Rendek (2009), which guarantees strict positive paths. However, like with any raw Monte-Carlo simulations, it can become computationally expensive and therefore, a variance reduction technique is highly recommended. In fact, the above explicit formulae for the defaultable bonds can be used as control variates or in other variance reduction methods for Monte Carlo simulations of the more general defaultable term structure models.

The paper is structured as follows. Section 2 provides the theoretical background for the modelling and pricing of real-world defaultable term structure models. In addition, we derive for certain volatility and intensity specifications tractable finite dimensional Markovian defaultable term structures. Section 3 introduces an alternative defaultable term structure model, with analytic expressions for prices of defaultable bonds. In Section 4, these analytic expressions for defaultable bond prices serve as control variates for an illustrative example on the effect of a variance reduction method in Monte Carlo simulations for pricing under a more general model. Section 5 concludes. 


\section{Real-World Pricing for a Defaultable Term Structure of Interest Rates}

\subsection{Modelling Traded Uncertainty}

On a filtered probability space $\left(\Omega, \mathcal{A}_{\bar{T}}, \underline{\mathcal{A}}, P\right), \bar{T} \in(0, \infty)$ with $\underline{\mathcal{A}}=\left(\mathcal{A}_{t}\right)_{t \in[0, \bar{T}]}$, satisfying the usual conditions, we model the continuous traded uncertainty as an $\underline{\mathcal{A}}$-adapted, $m$-dimensional Wiener process $W=\left\{W_{t}=\left(W_{t}^{1}, \ldots, W_{t}^{m}\right)^{\top}, t \in\right.$ $[0, \bar{T}]\}$. The event driven traded uncertainty is modelled by a Poisson random measure $p$, which is defined below. Given a mark space $(\mathcal{E}, \mathrm{B}(\mathcal{E}))$, with $\mathcal{E} \subseteq$ $\mathbb{R} \backslash\{0\}$, we define on $\mathcal{E} \times[0, \bar{T}]$ an $\underline{\mathcal{A}}$-adapted Poisson random measure $p(d v, d t)$ characterised by a time-varying intensity measure $\phi(d v, t) d t$. We assume almost surely finite total intensity $\lambda_{t}=\phi(\mathcal{E}, t)<\infty, t \in[0, \infty)$. Note that we allow the intensity measure $\phi(d v, t) d t$, and thus the total intensity $\lambda_{t}$, to be stochastic. Here $p=\left\{p_{t}:=p(\mathcal{E} \times[0, t]), t \in[0, \bar{T}]\right\}$ represents a stochastic process that counts the total number of jumps, that is modelled events occurring in the time interval $[0, t]$. The Poisson random measure $p(d v, d t)$ generates a sequence of pairs $\left\{\left(\tau_{i}, v_{i}\right), i \in\left\{1,2, \ldots, p_{\bar{T}}\right\}\right\}$, where $\left\{\tau_{i}: \Omega \rightarrow \mathbb{R}_{+}, i \in\left\{1,2, \ldots, p_{\bar{T}}\right\}\right\}$ is the sequence of jump times of the Poisson process $p$ and $\left\{v_{i}: \Omega \rightarrow \mathcal{E}, i \in\left\{1,2, \ldots, p_{\bar{T}}\right\}\right\}$ is the corresponding sequence of independent, identically distributed marks $v_{i}$ with probability density $\frac{\phi(d v, t)}{\lambda_{t}}$. One can interpret $\tau_{i}$ as the time of the $i^{t h}$ event and the mark $v_{i}$ as its magnitude. By compensating the Poisson measure we obtain the jump martingale measure $q(d v, d t)=p(d v, d t)-\phi(d v, t) d t$.

Additionally, we will assume that the drift, diffusion and jump coefficients of the factor processes driving the market dynamics to be predictable and regular enough such that the corresponding system of SDEs admits a unique strong solution and the manipulations we perform are possible. It will be not necessary to describe the primary securities of the market in detail. What is essentially needed for term structure modelling is the characterization of the GOP and the savings account.

\subsection{Growth Optimal Portfolio with Default}

The GOP is defined as the portfolio which maximises the expected logarithm of terminal wealth for all times $t \in[0, \bar{T}]$, see Kelly (1956), Karatzas and Shreve (1998) and Platen (2002). In our continuous time setting, the existence of a GOP implies no arbitrage in the strong sense of Platen (2002). Christensen and Platen (2005) consider a general jump-diffusion setting with stochastic jump sizes and obtain a generalised GOP, which we use here as our basis.

We denote the market prices of Wiener process risk by the predictable vector process $\Theta=\left\{\theta_{t}=\left(\theta_{t}^{1}, \theta_{t}^{2}, \ldots, \theta_{t}^{m}\right)^{\top}, t \in[0, \bar{T}]\right\}$, and the density of the market price of jump risk by the predictable and bounded process $\psi(v)=\{\psi(v, t), t \in$ $[0, \bar{T}]\}$, such that $\psi(v, t)<1$ for $(v, t) \in \mathcal{E} \times[0, \bar{T}]$. In addition, we consider the 
predictable default-free short rate process $r=\left\{r_{t}, t \in[0, \bar{T}]\right\}$. Then the unique generalised GOP, $S_{t}^{\delta_{*}}$, satisfies the SDE

$$
\begin{aligned}
d S_{t}^{\delta_{*}}=S_{t-}^{\delta_{*}} & \left(r_{t} d t\right. \\
& +\sum_{i=1}^{m} \theta_{t}^{i}\left(\theta_{t}^{i} d t+d W_{t}^{i}\right) \\
& \left.+\int_{\mathcal{E}} \frac{\psi(v, t)}{1-\psi(v, t)}(\psi(v, t) \phi(d v, t) d t+q(d v, d t))\right),
\end{aligned}
$$

for all $t \in[0, \bar{T}]$, with $S_{0}^{\delta_{*}}=1$, see Christensen and Platen (2005). Note that the dynamics of the GOP are determined solely by the default-free short rate $r_{t}$, the vector of market prices of Wiener process risk $\theta_{t}$ and the density of the market price of jump risk $\psi(v, t)$. Moreover, the total risk premium of the GOP at time $t \in[0, T]$ is given by

$$
\vartheta_{t}^{S}=\theta_{t}^{\top} \theta_{t}+\int_{\mathcal{E}} \frac{(\psi(v, t))^{2}}{1-\psi(v, t)} \phi(d v, t)
$$

From the SDE (2.1) we obtain that at the $i^{\text {th }}$ jump time $\tau_{i}$ of the Poisson jump measure we have

$$
S_{\tau_{i}}^{\delta_{*}}-S_{\tau_{i}-}^{\delta_{*}}=S_{\tau_{i}-}^{\delta_{*}} \frac{\psi\left(v_{i}, \tau_{i}-\right)}{1-\psi\left(v_{i}, \tau_{i}-\right)} .
$$

If we assume that the GOP is observable, then the density of the market price of jump risk can be observed in terms of the GOP values before and after jump times as

$$
\psi\left(v_{i}, \tau_{i}-\right)=1-\frac{S_{\tau_{i}-}^{\delta_{*}}}{S_{\tau_{i}}^{\delta_{*}}} .
$$

This gives access to the estimation and calibration of the function $\psi(\cdot, \cdot)$, which is crucial for realistic modelling. Note also that the volatility of the GOP, $\left|\theta_{t}\right|=$ $\sqrt{\sum_{i=1}^{m}\left(\theta_{t}^{i}\right)^{2}}$ is observable. However, the drift of a process is usually very difficult to estimate. Fortunately, such estimation is not necessary here since in (2.1), the drift depends on the observable short rate, GOP volatility, market price of jump risk density and jump intensity.

\subsection{Real-World Pricing}

In the following we will choose the GOP as numeraire or benchmark and will use the wording benchmarked when a price or value is expressed in units of the GOP. It has been shown in Platen and Heath (2006) that any nonnegative portfolio when expressed in units of the GOP forms an $(\underline{\mathcal{A}}, P)$-supermartingale, and in the set of all supermartingale price processes that match a given future payoff, the martingale is the one with the minimal value. Furthermore, we call a price 
process fair when it forms a martingale when benchmarked. Therefore, by requesting benchmarked derivative prices to be fair, the pricing of derivatives is performed under the real-world probability measure with the GOP as numeraire. Due to the supermartingale property of all benchmarked nonnegative portfolios, fair portfolios are minimal among those that replicate the same payoff. This yields the following concept of real-world pricing, see Long (1990) and Platen and Heath (2006).

Definition 2.1 For $T \in[0, \bar{T}]$ assume that $H_{T}$ is an $\mathcal{A}_{T}$-measurable contingent claim delivered at maturity $T$, which satisfies

$$
E\left(\frac{H_{T}}{S_{T}^{\delta_{*}}}\right)<\infty \text { almost surely. }
$$

Then the fair price process $V_{H_{T}}=\left\{V_{H_{T}}(t), t \in[0, T]\right\}$ of $H_{T}$ is given by the real-world pricing formula

$$
V_{H_{T}}(t)=S_{t}^{\delta_{*}} E\left(\frac{H_{T}}{S_{T}^{\delta_{*}}} \mid \mathcal{A}_{t}\right)
$$

for all $t \in[0, T]$.

Real-world pricing is performed by using in the evaluation the real-world expectation. Therefore, no change of probability measure is required. If an equivalent risk-neutral probability measure exists in a complete market, then the real-world pricing formula (2.6) can be shown to coincide with the risk-neutral one, see Platen and Heath (2006). Real-world pricing generalizes risk-neutral pricing and can be applied also under models which do not admit an equivalent risk-neutral probability measure, as will be demonstrated in Section 3.

\subsubsection{Real-World Defaultable Zero-Coupon Bond Dynamics}

Let $P^{d}(t, T)$ be the price at time $t \in[0, T]$ of a defaultable zero-coupon bond with maturity $T \in[0, \bar{T}]$. We assume fractional recovery upon default, adapting the setup of Schönbucher (1998). Typically, the fractional recovery arises as a result of restructuring and some reduction of the notional in case of default. Defaults can occur at jump times $\tau_{i} \leq T$. At each default time $\tau_{i}$, the fractional loss quota $v_{i}$ of the bond price is drawn from $\mathcal{E}=(0,1]$. We denote by $\overline{\mathcal{Q}}_{t}$ the reduction on the bond face value due to defaults until time $t$. Note that this approach allows for multiple defaults. At maturity $T$, the defaultable bond process $P^{d}(\cdot, T)$ pays out

$$
\overline{\mathcal{Q}}_{T}:=\prod_{i: \tau_{i} \leq T}\left(1-v_{i}\right)
$$


the remaining face value after all fractional losses. The face value $\overline{\mathcal{Q}}_{t}$ is assumed to be the solution of the SDE

$$
d \overline{\mathcal{Q}}_{t}=-\overline{\mathcal{Q}}_{t-} \int_{0}^{1} v p(d v, d t)
$$

for $t \in[0, T]$, subject to the initial condition $\overline{\mathcal{Q}}_{0}=1$. By using (2.6) and assuming $E\left(\left(S_{T}^{\delta_{*}}\right)^{-1}\right)<\infty$, the real-world price at time $t \in[0, T]$ for the defaultable zerocoupon bond with maturity $T$ is

$$
P^{d}(t, T)=S_{t}^{\delta_{*}} E\left(\frac{\overline{\mathcal{Q}}_{T}}{S_{T}^{\delta_{*}}} \mid \mathcal{A}_{t}\right)
$$

for $t \in[0, T], T \in[0, \bar{T}]$. Relationship (2.9) guarantees that the benchmarked defaultable zero-coupon bond, denoted as

$$
\hat{P}^{d}(t, T)=\frac{P^{d}(t, T)}{S_{t}^{\delta_{*}}}
$$

is an $(\underline{\mathcal{A}}, P)$-martingale and thus, fair. Therefore, it satisfies a driftless SDE of the form

$$
d \hat{P}^{d}(t, T)=-\hat{P}^{d}(t-, T)\left(\sum_{i=1}^{m} \hat{\sigma}^{i}(t, T) d W_{t}^{i}+\int_{0}^{1} \hat{\beta}(v, t, T) q(d v, d t)\right)
$$

Here $\hat{\sigma}^{i}(\cdot, T)$, for $i \in\{1,2, \ldots, m\}$, and $\hat{\beta}(v, \cdot, T)$ are predictable stochastic processes modelling the benchmarked defaultable bond volatilities and jump coefficient, respectively. By (2.1), (2.10), (2.11) and Itô's formula we obtain the dynamics of the defaultable zero-coupon bond price as

$$
\begin{aligned}
d P^{d}(t, T)=P^{d}(t-, T) & {\left[\left(r_{t}+\sum_{i=1}^{m} \sigma^{i}(t, T) \theta_{t}^{i}+\int_{0}^{1} \beta(v, t, T)(\psi(v, t)-1) \phi(d v, t)\right) d t\right.} \\
& \left.+\sum_{i=1}^{m} \sigma^{i}(t, T) d W_{t}^{i}+\int_{0}^{1} \beta(v, t, T) p(d v, d t)\right]
\end{aligned}
$$

for all $t \in[0, T]$, with defaultable bond volatilities

$$
\sigma^{i}(t, T)=\theta_{t}^{i}-\hat{\sigma}^{i}(t, T)
$$

$i \in\{1,2, \ldots, m\}$, and jump coefficient

$$
\beta(v, t, T)=\frac{\psi(v, t)-\hat{\beta}(v, t, T)}{1-\psi(v, t)} .
$$

Note that at each default time $\tau_{i}$, the relative change in the bond price

$$
\frac{P^{d}\left(\tau_{i}, T\right)-P^{d}\left(\tau_{i}-, T\right)}{P^{d}\left(\tau_{i}-, T\right)}=\beta\left(v_{i}, \tau_{i}, T\right)
$$


can be decomposed into two distinct effects. First, after default restructuring of the defaulted obligor's business takes place, and there is a reduction of the claims of the bond holders, which leads to a fractional loss equal to $v_{i}$ in the bond price. Second, additionally, the market's valuation of the bond can change, see Schönbucher (1998). One can model both effects by decomposing the jump coefficient of the defaultable bond as

$$
\beta(v, t, T)=\beta_{M}(v, t, T)-v,
$$

where $\beta_{M}(v, t, T)$ reflects the jump due to the change in the market valuation.

\subsubsection{Real-World Defaultable Forward Rate Dynamics}

We define the instantaneous defaultable forward rate $f^{d}(t, T)$ at time $t \in[0, T]$, with $T \in[0, \bar{T}]$, as

$$
f^{d}(t, T):=-\frac{\partial}{\partial T} \ln \left(P^{d}(t, T)\right)
$$

We also define the predictable defaultable short rate process as $r^{d}=\left\{r_{t}^{d}:=\right.$ $\left.f^{d}(t, t), t \in[0, T]\right\}$. Then by taking into account the face value reduction $\overline{\mathcal{Q}}_{t}$ of the bond at time $t$, the value of the defaultable bond $P^{d}(t, T)$ can be expressed as

$$
P^{d}(t, T)=\exp \left(-\int_{t}^{T} f^{d}(t, s) d s\right) \overline{\mathcal{Q}}_{t} .
$$

Note that by relationships (2.10) and (2.17) the defaultable forward rate can be equivalently expressed in terms of the benchmarked defaultable bond as

$$
f^{d}(t, T)=-\frac{\partial}{\partial T} \ln \left(\hat{P}^{d}(t, T)\right) .
$$

As shown in Appendix A, this yields the following result, where $\hat{\sigma}^{i}(t, T), i \in$ $\{1,2, \ldots, m\}$, and $\hat{\beta}(v, t, T)$ are defined in (2.11).

Proposition 2.2 The real-world dynamics of the instantaneous defaultable forward rate are given by

$$
d f^{d}(t, T)=\mu_{d}(t, T) d t+\sum_{i=1}^{m} \sigma_{d}^{i}(t, T) d W_{t}^{i}+\int_{0}^{1} \beta_{d}(v, t, T) p(d v, d t)
$$

where

$$
\begin{aligned}
\mu_{d}(t, T)= & \sum_{i=1}^{m} \sigma_{d}^{i}(t, T)\left(\int_{t}^{T} \sigma_{d}^{i}(t, s) d s+\theta_{t}^{i}\right) \\
& -\int_{0}^{1} \beta_{d}(v, t, T) \exp \left(-\int_{t}^{T} \beta_{d}(v, t, s) d s\right)(1-\psi(v, t))(1-v) \phi(d v, t),
\end{aligned}
$$


and

$$
\begin{array}{r}
\sigma_{d}^{i}(t, T)=\frac{\partial}{\partial T} \hat{\sigma}^{i}(t, T), \\
\beta_{d}(v, t, T)=\frac{\frac{\partial}{\partial T} \hat{\beta}(v, t, T)}{1-\hat{\beta}(v, t, T)} .
\end{array}
$$

The defaultable forward rate drift restriction (2.21) has a similar structure to the HJM restriction described in Schönbucher (2003), which was derived under some risk-neutral probability measure. However, the drift restriction (2.21) holds under the real-world probability measure under which $W_{t}^{1}, \ldots, W_{t}^{m}$ are Wiener processes and $p(\cdot, \cdot)$ is a Poisson measure with intensity measure $\phi(d v, t)$. This provides an advantage of the benchmark approach over the risk-neutral approach concerning the estimation of default intensities and probabilities. Real-world default probabilities can be estimated using historical data and credit spreads, or by economic reasoning. In contrast, risk-neutral default probabilities can be obtained only by using some model applied to observed credit spreads. As previously discussed, these spreads can be way out from reality if one relies on relative pricing. By using the real-world probability measure for calibration and pricing, the benchmark approach avoids the challenge of estimating hypothetical riskneutral probabilities of default from credit derivatives in the market or by other means. Albanese and Chen (2005) provide an interesting study of this controversial problem. As we have already pointed out in the introduction, we perform a type of absolute pricing, whereas the standard approach yields relative prices.

Note that by (2.14), (2.16) and (2.23), the defaultable forward rate jump coefficient can be expressed as

$$
\beta_{d}(v, t, T)=\frac{\frac{\partial}{\partial T} \beta_{M}(v, t, T)}{v-1-\beta_{M}(v, t, T)} .
$$

This shows that if in our setting the jumps in the defaultable bond price due to changes in the market valuation at default do not depend on the maturity $T$ of the bond, this means $\beta_{M}(v, t, T)=\beta_{M}(v, t)$, then we obtain continuous defaultable forward rates.

In Appendix B, we derive a relationship for the short rate spread in terms of the real-world intensity measure and the market price of jump risk as

$$
r_{t}^{d}-r_{t}=\int_{0}^{1}(1-\psi(v, t)) v \phi(d v, t) .
$$

If an equivalent risk-neutral probability measure $\Lambda$ exists, then the risk-neutral intensity measure $\phi_{\Lambda}(d v, t)$ can be expressed as $\phi_{\Lambda}(d v, t)=(1-\psi(v, t)) \phi(d v, t)$ and the spread $(2.25)$ reduces to

$$
r_{t}^{d}-r_{t}=\int_{0}^{1} v \phi_{\Lambda}(d v, t),
$$


which is equivalent to a result in Schönbucher (1998). However, there is no need to restrict our modelling by requiring the existence of an equivalent risk-neutral probability measure.

\subsection{Finite Dimensional Markovian Term Structures}

When keeping the framework on the current general level, one faces certain mathematical challenges concerning the consistency of the model in the context of enlargement of filtration, see Elliott, Jeanblanc, and Yor (2000). In practice one needs tractable models that, in principle, lead to Markovian model structures, which usually avoid consistency problems of this kind. Otherwise, the need for the description and storage of the entire history of the market would create unsolvable practical obstacles. However, even in Markovian settings, one has to be parsimonious with the number of factors and parameters in the model in order to keep the dimensionality of the problem manageable and to allow a proper fitting of market data.

Though very flexible, HJM term structure models are, in general, non-Markovian. It has been demonstrated in Chiarella, Schlögl, and Nikitopoulos (2007) that, under the existence of an equivalent risk-neutral probability measure, specific forward rate volatility structures can produce finite dimensional Markovian defaultable HJM models which are computationally tractable. It will be shown next that certain forward rate volatility specifications will lead to tractable finite dimensional Markovian dynamics for the defaultable interest rate term structure under the real-world probability measure without relying on any risk-neutral framework.

Assumption 2.3 For $T \in(0, \bar{T}]$ and $i \in\{1, \ldots, m\}$, the diffusion coefficients (2.22) of the defaultable forward rate are of the form

$$
\sigma_{d}^{i}(t, T)=\bar{\sigma}_{d}^{i}\left(t, \mathrm{~F}_{t}\right) e^{-\int_{t}^{T} k_{\sigma}^{i}(s) d s},
$$

and the jump coefficient (2.23) is of the form

$$
\beta_{d}(v, t, T)=\bar{\beta}_{d}(v, t) e^{-\int_{t}^{T} k_{\beta}(s) d s},
$$

where $k_{\sigma}^{i}(t), k_{\beta}(t)$ are deterministic functions of time, integrable in [0,T]; and $\bar{\sigma}_{d}^{i}=\left\{\bar{\sigma}_{d}^{i}\left(t, \mathrm{~F}_{t}\right), t \in[0, T]\right\}$ and $\bar{\beta}_{d}=\left\{\bar{\beta}_{d}(v, t),(v, t) \in(0,1] \times[0, T]\right.$, $\}$ characterise well-defined functions, with $\mathrm{F}_{t}=\left(r_{t}^{d}, f^{d}\left(t, T_{1}\right), \ldots, f^{d}\left(t, T_{z}\right)\right)^{\top}$, for $z \in \mathbb{N}=$ $\{1,2,3, \ldots\}$ and $t<T_{1}<\ldots<T_{z}$. In addition, the density of the market price of jump risk $\psi=\{\psi(v, t),(v, t) \in(0,1] \times[0, T]\}$ and the jump intensity measure $\phi(d v, t), t \in[0, T]$, are deterministic.

The key property of the proposed volatility structure is the separability of the time dependent component from the maturity dependent component. The resulting Markovian dynamics of the defaultable short rate are derived in Appendix $\mathrm{C}$ and are summarized in the following proposition. 
Proposition 2.4 Under Assumption 2.3 the real-world dynamics of the defaultable short rate are given by

$$
\begin{aligned}
d r_{t}^{d}= & {\left[\xi_{t}+\mathcal{E}_{\beta}(t)+\sum_{i=1}^{m} \mathcal{E}_{\sigma}^{i}(t)-\sum_{i=2}^{m}\left(k_{\sigma}^{i}(t)-k_{\sigma}^{1}(t)\right) \mathcal{D}_{\sigma}^{i}(t)-\left(k_{\beta}(t)-k_{\sigma}^{1}(t)\right) \mathcal{D}_{\beta}(t)\right.} \\
& \left.+\sum_{i=1}^{m} \bar{\sigma}_{d}^{i}\left(t, \mathrm{~F}_{t}\right) \theta_{t}^{i}-k_{\sigma}^{1}(t) r_{t}\right] d t+\sum_{i=1}^{m} \bar{\sigma}_{d}^{i}\left(t, \mathrm{~F}_{t}\right) d W_{t}^{i}+\int_{0}^{1} \bar{\beta}_{d}(v, t) p(d v, d t)
\end{aligned}
$$

with the state variables $\mathcal{E}_{\sigma}^{i}(t), \mathcal{D}_{\sigma}^{i}(t), \mathcal{D}_{\beta}(t)$ defined as

$$
\begin{aligned}
\mathcal{E}_{\sigma}^{i}(t)= & \int_{0}^{t}\left(\sigma_{d}^{i}(u, t)\right)^{2} d u \\
\mathcal{D}_{\sigma}^{i}(t)= & \int_{0}^{t} \sigma_{d}^{i}(u, t) \int_{u}^{t} \sigma_{d}^{i}(u, s) d s d u+\int_{0}^{t} \sigma_{d}^{i}(u, t)\left(d W_{u}^{i}+\theta_{u}^{i} d u\right) \\
\mathcal{D}_{\beta}(t)=- & \int_{0}^{t} \int_{0}^{1} \beta_{d}(v, u, t) e^{-\int_{u}^{t} \beta_{d}(v, u, s) d s}(1-v)(1-\psi(v, u)) \phi(d v, t) d u \\
& +\int_{0}^{t} \int_{0}^{1} \beta_{d}(v, u, t) p(d v, d u)
\end{aligned}
$$

respectively, and the time varying coefficients $\mathcal{E}_{\beta}(t)$ and $\xi_{t}$ are determined by

$$
\begin{aligned}
\mathcal{E}_{\beta}(t)= & \int_{0}^{t} \int_{0}^{1} \beta_{d}(v, u, t)^{2} e^{-\int_{u}^{t} \beta_{d}(v, u, s) d s}(1-v)(1-\psi(v, u)) \phi(d v, t) d u \\
& -\int_{0}^{1} \bar{\beta}_{d}(v, t)(1-v)(1-\psi(v, t)) \phi(d v, t), \\
\xi_{t}= & \left.\frac{\partial}{\partial T} f^{d}(0, T)\right|_{T=t}+k_{\sigma}^{1}(t) f^{d}(0, t),
\end{aligned}
$$

respectively.

The stochastic quantities $\mathcal{E}_{\sigma}^{i}(t), \mathcal{D}_{\sigma}^{i}(t)$ and $\mathcal{D}_{\beta}(t)$ form state variables of linear mean reverting SDEs, as the following proposition demonstrates.

Proposition 2.5 Under Assumption 2.3, the stochastic quantities $\mathcal{E}_{\sigma}^{i}(t), \mathcal{D}_{\sigma}^{i}(t)$ and $\mathcal{D}_{\beta}^{i}(t)$ satisfy the SDEs,

$$
\begin{gathered}
d \mathcal{E}_{\sigma}^{i}(t)=\left[\left(\bar{\sigma}_{d}^{i}\left(t, \mathrm{~F}_{t}\right)\right)^{2}-2 \kappa_{\sigma}^{i}(t) \mathcal{E}_{\sigma}^{i}(t)\right] d t \\
d \mathcal{D}_{\sigma}^{i}(t)=\left[\mathcal{E}_{\sigma}^{i}(t)-\kappa_{\sigma}^{i}(t) \mathcal{D}_{\sigma}^{i}(t)\right] d t+\bar{\sigma}_{d}^{i}\left(t, \mathrm{~F}_{t}\right)\left(d W_{t}^{i}+\theta_{t}^{i} d t\right),
\end{gathered}
$$

and

$$
d \mathcal{D}_{\beta}(t)=\left[\mathcal{E}_{\beta}(t)-\kappa_{\beta}(t) \mathcal{D}_{\beta}(t)\right] d t+\int_{0}^{1} \bar{\beta}_{d}(v, t) p(d v, d t)
$$


Proof. Taking the differentials of the stochastic quantities (2.30), (2.31) and (2.32), the above equations are obtained.

Additionally, to obtain a closed Markovian system, the stochastic market prices of Wiener process risk $\theta_{t}^{i}$ should satisfy an SDE with coefficients depending on the state variables of that system. Thus, the finite dimensional Markovian system with the state vector $\left(r_{t}^{d}, f^{d}\left(t, T_{1}\right), \ldots, f^{d}\left(t, T_{z}\right), \theta_{t}^{1}, \mathcal{E}_{\sigma}^{1}(t), \mathcal{D}_{\sigma}^{1}(t), \ldots, \theta_{t}^{m}, \mathcal{E}_{\sigma}^{m}(t)\right.$, $\left.\mathcal{D}_{\sigma}^{m}(t), \mathcal{D}_{\beta}(t)\right)^{\top}$, for $z, m \in \mathbb{N}$ determines the short rate dynamics (2.29). Recall that the state variables $f^{d}\left(t, T_{k}\right), k \in(1,2, \ldots, z)$, satisfy the $\operatorname{SDE}(2.20)$ under the volatility structure of Assumption 2.3. Alternatively, the market prices of Wiener process risk can be modelled by an additional set of state variables, as these are the only state variables driving the dynamics of the discounted GOP.

Assumption 2.3 imposes the restriction of a deterministic market price of jump risk $\psi(v, t)$ and a deterministic jump intensity $\phi(d v, t)$. A general specification which would allow stochastic market price of jump risk and stochastic jump intensity is feasible, but would lead to Markovian structures only in the case of constant jump coefficients. For a jump coefficient of the form (2.28) an approximate Markovianisation can be achieved along the lines of Chiarella, Schlögl, and Nikitopoulos (2007).

\section{Tractable Defaultable Multi-Factor Models}

A class of parsimonious defaultable multi-factor models will be presented next. These models provide computationally tractable formulas for defaultable zerocoupon bonds. The numerical evaluation is conveniently performed in a multifactor setting of the following kind which represents a special case of the previous class of Markovian models.

\subsection{Real-World Default-Adjusted Short Rate}

We start from a $d$-dimensional factor process whose dynamics are described by the SDE

$$
d X_{t}=a\left(t, X_{t}\right) d t+b\left(t, X_{t}\right) d W_{t},
$$

where $W$ denotes the $m$-dimensional standard Wiener process representing the continuous traded underlying. In this section we assume, for simplicity, that the jump process generating defaults is a compound Cox process, also called compound doubly stochastic Poisson process, see for instance Duffie (2005), with stochastic intensity process $\lambda=\left\{\lambda_{t}, t \in[0, \infty)\right\}$. We suppose that the $\sigma$-algebra generated by the factor process $X=\left\{X_{t}, t \in[0, T]\right\}$ defines the filtration $\underline{\mathcal{G}}=$ $\left(\mathcal{G}_{t}\right)_{t \in[0, \infty)} \subset\left(\mathcal{A}_{t}\right)_{t \in[0, \infty)}=\underline{\mathcal{A}}$. Moreover, we suppose that the default-free interest rate $r_{t}$, the discounted GOP $\bar{S}_{t}^{\delta_{*}}=\frac{S_{t}^{\delta_{*}}}{S_{t}^{0}}$ and the intensity process $\lambda_{t}=\lambda\left(t, X_{t}\right)$ can 
be expressed as measurable nonnegative functions of time $t$ and $X_{t}$. Therefore, they are adapted to $\underline{\mathcal{G}}=\left(\mathcal{G}_{t}\right)_{t \in[0, \infty)}$. The jump sizes $v_{i}$, which represent the fractional loss quota, are independent identically distributed random variables with mean $E(v)=m_{v}$ and are also assumed to be independent of the factor process $X$. Then, at any time $t \in[0, T]$ the compound Cox process conditioned on the $\sigma$-algebra $\mathcal{G}_{T} \vee \mathcal{A}_{t}$, generated by the events in $\mathcal{G}_{T} \cup \mathcal{A}_{t}$, is up to time $T$ an inhomogeneous compound Poisson process with intensity $\lambda=\left\{\lambda\left(t, X_{t}\right), t \in\right.$ $[0, T]\}$. This setting is analogous to the approach used in Schönbucher (2003), however, we do not perform any risk neutral measure change and allow more general market dynamics.

The defaultable zero-coupon bond $P^{d}(t, T)$, see (2.9), can be expressed in terms of the discounted GOP $\bar{S}_{t}^{\delta_{*}}$, the default-free savings account

$$
S_{t}^{0}=\exp \left\{\int_{0}^{t} r_{s} d s\right\}
$$

and the remaining face value after all fractional losses $\overline{\mathcal{Q}}_{T}$, see $(2.7)$, as

$$
P^{d}(t, T)=E\left(\frac{S_{t}^{\delta_{*}}}{S_{T}^{\delta_{*}}} \overline{\mathcal{Q}}_{T} \mid \mathcal{A}_{t}\right)=E\left(\frac{\bar{S}_{t}^{\delta_{*}}}{\bar{S}_{T}^{\delta_{*}}} \frac{S_{T}^{0}}{S_{t}^{0}} \overline{\mathcal{Q}}_{T} \mid \mathcal{A}_{t}\right)
$$

for $t \in[0, T], T \in[0, \bar{T}]$. By conditioning on the $\sigma$-algebra $\mathcal{G}_{T} \vee \mathcal{A}_{t}$, one obtains

$$
\begin{aligned}
P^{d}(t, T) & =\overline{\mathcal{Q}}_{t} E\left(\frac{\bar{S}_{t}^{\delta_{*}}}{\bar{S}_{T}^{\delta_{*}}} \frac{S_{T}^{0}}{S_{t}^{0}} \prod_{\tau_{i} \in(t, T]}\left(1-v_{i}\right) \mid \mathcal{A}_{t}\right) \\
& =\overline{\mathcal{Q}}_{t} E\left(\frac{\bar{S}_{t}^{\delta_{*}}}{\bar{S}_{T}^{\delta_{*}}} \exp \left\{-\int_{t}^{T} r_{s} d s\right\} E\left(\prod_{\tau_{i} \in(t, T]}\left(1-v_{i}\right) \mid \mathcal{G}_{T} \vee \mathcal{A}_{t}\right) \mid \mathcal{A}_{t}\right) \\
& =\overline{\mathcal{Q}}_{t} E\left(\frac{\bar{S}_{t}^{\delta_{*}}}{\bar{S}_{T}^{\delta_{*}}} \exp \left\{-\int_{t}^{T}\left(r_{s}+\lambda_{s} m_{v}\right) d s\right\} \mid \mathcal{A}_{t}\right) .
\end{aligned}
$$

Let us also consider the price $V(t, T)$, at time $t \in[0, T]$, of a defaultable contingent claim $H_{T}=\overline{\mathcal{Q}}_{T} \tilde{H}\left(X_{T}\right)$. This claim has the following payoff features: if there are no defaults before time $T$, then the contingent claim pays the $\mathcal{G}_{T}$-adapted payoff $\tilde{H}\left(X_{T}\right)$ at time $T$. Otherwise, it pays the payoff $\overline{\mathcal{Q}}_{T} \tilde{H}\left(X_{T}\right)$, which depends on the recovery rates. Similar to (3.4) one can derive the representation

$$
V(t, T)=\overline{\mathcal{Q}}_{t} \bar{S}_{t}^{\delta_{*}} E\left(\exp \left\{-\int_{t}^{T}\left(r_{s}+\lambda_{s} m_{v}\right) d s\right\} \frac{\tilde{H}\left(X_{T}\right)}{\bar{S}_{T}^{\delta_{*}}} \mid \mathcal{A}_{t}\right) .
$$

This provides a similar representation as in Duffie and Singleton (1999), however, here obtained under the real-world probability measure. Furthermore, we avoid any problems that may result from defining the above filtration improperly, see 
Elliott, Jeanblanc, and Yor (2000). In particular, in this setting the price of a defaultable contingent claim can be represented as a conditional expectation under the real-world probability measure of the appropriately discounted benchmarked payoff of an equivalent default-free contingent claim. The appropriate discount factor is given by an exponential involving the default-free short rate $r_{t}$ plus the real-world mean-loss rate $\lambda_{t} m_{v}$. Note that in the case of a compound Cox process, as considered here, the spread (2.26) reduces to the expression

$$
r_{t}^{d}-r_{t}=\lambda_{t}\left(m_{v}-E(\psi(t) v)\right)
$$

Therefore, the real-world default-adjusted short rate $r_{t}+\lambda_{t} m_{v}$ does not coincide with the defaultable short rate $r_{t}^{d}$. Of course, if an equivalent risk-neutral probability measure exists, then one recovers the result of Duffie and Singleton (1999). However, in reality the discounted proxy for the GOP, as the S\&P 500 accumulation index, has stochastic volatility and its inverse appears to follow a strict supermartingale rather than a martingale. This suggests that some more general modelling approach is needed than the one provided by the risk neutral approach, which requires the inverse of the savings account discounted GOP to form a martingale.

It is still a numerical challenge to calculate credit derivative prices efficiently for the general class of models given above. The most flexible numerical method seems still to be Monte Carlo simulation, in particular, when more than two factors are involved. To apply this method efficiently, variance reduction techniques are strongly recommended, see Kloeden and Platen (1999). For the contruction of variance reduced estimators it is very important to have some explicit solutions available for at least some special models. We present next a class of models that provide tractable solutions, suitable for variance reduction methods.

\subsection{Explicit Formula for Defaultable Bonds}

In our special class of models we assume independence between the discounted GOP and the default-adjusted short rate. Then the defaultable zero-coupon bond price $P^{d}(t, T)$ in (3.4) is obtained as the product

$$
P^{d}(t, T)=\overline{\mathcal{Q}}_{t} E\left(\frac{\bar{S}_{t}^{\delta_{*}}}{\bar{S}_{T}^{\delta_{*}}} \mid \mathcal{A}_{t}\right) E\left(\exp \left\{-\int_{t}^{T}\left(r_{s}+\lambda_{s} m_{v}\right) d s\right\} \mid \mathcal{A}_{t}\right) .
$$

To explicitly evaluate these two expectations we consider a specification for a class of three factor Markovian models, where the factors are the discounted GOP $\bar{S}^{\delta_{*}}=\left\{\bar{S}_{t}^{\delta_{*}}=\frac{\bar{S}_{t}^{\delta_{*}}}{S_{t}^{0}}, t \in[0, \infty)\right\}$, the short rate $r=\left\{r_{t}, t \in[0, \infty)\right\}$ and the jump intensity $\lambda=\left\{\lambda_{t}, t \in[0, \infty)\right\}$.

Recall that $S_{t}^{0}$ denotes the default free savings account (3.2), which continuously accrues the short rate $r_{t}$. For simplicity, assume that the GOP follows continuous 
dynamics. In this case, due to the continuity of the GOP, the market price of jump risk is zero. Thus, the GOP follows the dynamics (2.1) with the specification $\psi(v, t)=0$. The discounted GOP dynamics are then given by

$$
d \bar{S}_{t}^{\delta_{*}}=\bar{S}_{t-}^{\delta_{*}} \sum_{i=1}^{m} \theta_{t}^{i}\left(\theta_{t}^{i} d t+d W_{t}^{i}\right)
$$

which solely depend on the total market price of risk $\left|\theta_{t}\right|=\sqrt{\sum_{i=1}^{m}\left(\theta_{t}^{i}\right)^{2}}$. The discounted GOP drift equals

$$
\alpha_{t}=\bar{S}_{t}^{\delta_{*}}\left|\theta_{t}\right|^{2}
$$

Thus, the total market price of risk can be expressed as

$$
\left|\theta_{t}\right|=\sqrt{\frac{\alpha_{t}}{\bar{S}_{t}^{\delta_{*}}}}
$$

By using (3.9) and (3.10) in (3.8), Platen and Heath (2006) show that the discounted GOP is a time transformed squared Bessel process. The stylised minimal market model, proposed in Platen (2001) and Platen (2002), emerges when specifying a deterministic time transformation. More precisely, we assume in this section that the discounted GOP drift $\alpha_{t}, t \in[0, T]$, has the form

$$
\alpha_{t}=\alpha_{0} \exp \{\eta t\} \text {. }
$$

Here $\eta>0$ is the constant net growth rate and $\alpha_{0}>0$ is an initial scaling parameter. The discounted GOP $\bar{S}_{t}^{\delta_{*}}$ is then a time transformed squared Bessel process of dimension four with a deterministic transformed time. By applying the explicitly known transition density of $\bar{S}_{t}^{\delta_{*}}$, the market price of risk contribution to the bond price, is obtained by the formula

$$
E\left(\frac{\bar{S}_{t}^{\delta_{*}}}{\bar{S}_{T}^{\delta_{*}}} \mid \mathcal{A}_{t}\right)=1-\exp \left\{-\frac{2 R(t, T) \bar{S}_{t}^{\delta_{*}}}{\alpha_{t}}\right\},
$$

with

$$
R(t, T)=\frac{\eta}{\exp \{\eta(T-t)\}-1}
$$

see Platen (2002).

The market price of the default-adjusted short rate contribution to the bond can be explicitly evaluated, for instance, for the affine term structure models derived in Bruti-Liberati, Nikitopoulos-Sklibosios, and Platen (2007). For the sake of simplicity, we restrict the special class of models to a deterministic default-free short rate. We thus overcome the issue of obtaining negative spreads arising when both the default-free short rate and the default-adjusted short rate are evolving stochastically. When pricing off-balance-sheet credit derivatives, for instance CDSs, default-free short rate risk has a secondary effect. Therefore, we avoid unnecessary complexity, while focusing on the important factor which is 
here the credit spread. The credit spread is then evolving stochastically if the intensity is modelled as a stochastic process. In this case we obtain the defaultadjusted short rate contribution to the defaultable zero-coupon bond, see (3.7), as

$$
E\left(\exp \left\{-\int_{t}^{T}\left(r_{s}+\lambda_{s} m_{v}\right) d s\right\} \mid \mathcal{A}_{t}\right)=e^{-\int_{t}^{T} r_{s} d s} E\left(e^{-m_{v} \int_{t}^{T} \lambda_{s} d s} \mid \mathcal{A}_{t}\right) .
$$

We can evaluate explicitly the expectation $E\left(e^{-m_{v} \int_{t}^{T} \lambda_{s} d s} \mid \mathcal{A}_{t}\right)$, by using the tractable solutions derived in Bruti-Liberati, Nikitopoulos-Sklibosios, and Platen (2007). By assuming that the stochastic jump intensity $\lambda_{t}$ follows the dynamics

$$
d \lambda_{t}=\kappa\left(\bar{\lambda}-\lambda_{t}\right) d t+\sigma \sqrt{\lambda_{t}} d \tilde{W}_{t}+d J_{t}
$$

where $\kappa, \bar{\lambda}, \sigma$ are positive constants, with $2 \kappa \bar{\lambda}>\sigma^{2}$, we directly obtain CIR type closed form solutions for the default-adjusted short rate contribution (3.13). Note that, the Wiener process $\tilde{W}_{t}$ is assumed to be independent of the driving Wiener processes $W_{t}^{i}, i \in\{1,2, \ldots, m\}$. Here $J=\left\{J_{t}, t \in[0, \infty)\right\}$ is a compound Poisson process with intensity $\lambda$ and exponentially distributed marks $v_{i}^{\lambda}$ with mean $1 / h$, independent of the Cox process generating defaults. Then by combining these results with expression (3.12), we arrive at the following analytic formula for the defaultable zero-coupon bond price (3.7)

$$
P_{\text {analytic }}^{d}(t, T)=\overline{\mathcal{Q}}_{t}\left(1-e^{-\frac{2 R(t, T) \bar{S}_{t}^{\delta_{*}}}{\alpha_{t}}}\right) e^{-\int_{t}^{T} r_{s} d s} e^{m_{v}\left[A(t, T)-B(t, T) \lambda_{t}\right]},
$$

where

$$
B(t, T)=\frac{L_{1}(T-t)}{L_{2}(T-t)}
$$

and

$$
\begin{aligned}
A(t, T) & =\frac{2 \kappa \bar{\lambda}}{\sigma^{2}} \ln \left(\frac{L_{3}(T-t)}{L_{2}(T-t)}\right) \\
& +\frac{\lambda h}{1+\kappa h-0.5 \sigma^{2} h^{2}} \ln \left(e^{-\frac{t}{h}} \frac{L_{1}(T-t)+h L_{2}(T-t)}{h L_{3}(T-t)}\right)
\end{aligned}
$$

with

$$
\begin{aligned}
& L_{1}(t)=2\left(e^{\varpi_{1} t}-1\right), \\
& L_{2}(t)=\varpi_{1}\left(e^{\varpi_{1} t}+1\right)+\kappa\left(e^{\varpi_{1} t}-1\right), \\
& L_{3}(t)=2 \varpi_{1} e^{\left(\varpi_{1}+\kappa\right) t / 2},
\end{aligned}
$$

and $\varpi_{1}=\sqrt{\kappa^{2}+2 \sigma^{2}}$, see Filipović (2001). 


\subsubsection{Defaultable Forward Rate}

Under the assumption of independence between the discounted GOP and the default-adjusted short rate, from definition (2.17) and (3.7), we can express the forward rate as the following sum

$$
f(t, T)=n(t, T)+\varrho(t, T) .
$$

The market price of risk contribution function $n(t, T)$, due to (3.12), is reduced to

$$
n(t, T):=-\frac{\partial}{\partial T} \ln \left[E\left(\frac{\bar{S}_{t}^{\delta_{*}}}{\bar{S}_{T}^{\delta_{*}}} \mid \mathcal{A}_{t}\right)\right]=\frac{2 R(t, T)(\eta+R(t, T))}{\left|\theta_{t}\right|^{2}\left(\exp \left(\frac{2 R(t, T)}{\left|\theta_{t}\right|^{2}}\right)-1\right)} .
$$

The default-adjusted short rate contribution function $\varrho(t, T)$ is given by

$$
\varrho(t, T):=-\frac{\partial}{\partial T} \ln \left[E\left(\exp \left\{-\int_{t}^{T}\left(r_{s}+\lambda_{s} m_{v}\right) d s\right\} \mid \mathcal{A}_{t}\right)\right] .
$$

Assuming the representation (3.13), which considers deterministic short rate $r_{t}$ and the dynamics (3.14) for the default intensity, the default-adjusted short rate contribution function $\varrho(t, T)$ is reduced to

$$
\varrho(t, T)=r_{T}-m_{v} \frac{\partial A(t, T)}{\partial T}+m_{v} \frac{\partial B(t, T)}{\partial T} \lambda_{t}
$$

where $A(t, T)$ and $B(t, T)$ are given by (3.17) and (3.16), respectively.

Platen (2005) investigates the properties of the market price of risk contribution function (3.20). It has been shown that for short maturities the market price of risk contribution function is practically zero while for longer maturities it approaches asymptotically the value of the net growth rate $\eta$. The empirical study of Matacz and Bouchaud (2000) shows flat spreads for short maturities and hump shaped spreads for longer maturities, which are consistent with the properties of the function (3.20).

Figure 3.1 plots the forward rate (3.19) as function of the maturity $T$ and the initial default intensity $\lambda_{0} \in[0.03,0.15]$. The net growth rate is $\eta=0.05$, the initial market price of risk is $\theta_{0}=0.2$. We have also used $\kappa=2$, and $\bar{\lambda}=0.05$, $\sigma=0.15, m_{v}=1$, and $h=0.2$. Furthermore, we assume that the short rate evolves as a deterministic function of time namely $d r_{t}=a\left(b-r_{t}\right) d t$, with $a=2$, $b=0.06$ and $r_{0}=0.08$. Thus $r_{t}=e^{-a t}\left(r_{0}-b\right)+b$. At the short end of the forward rate curve the default-adjusted short rate dominates the market price of risk contribution, while at the long end the reverse holds true.

\section{Monte Carlo Simulations}

When dependence between the discounted GOP and the default-adjusted short rate is assumed, one can still evaluate the bond price using numerical techniques 


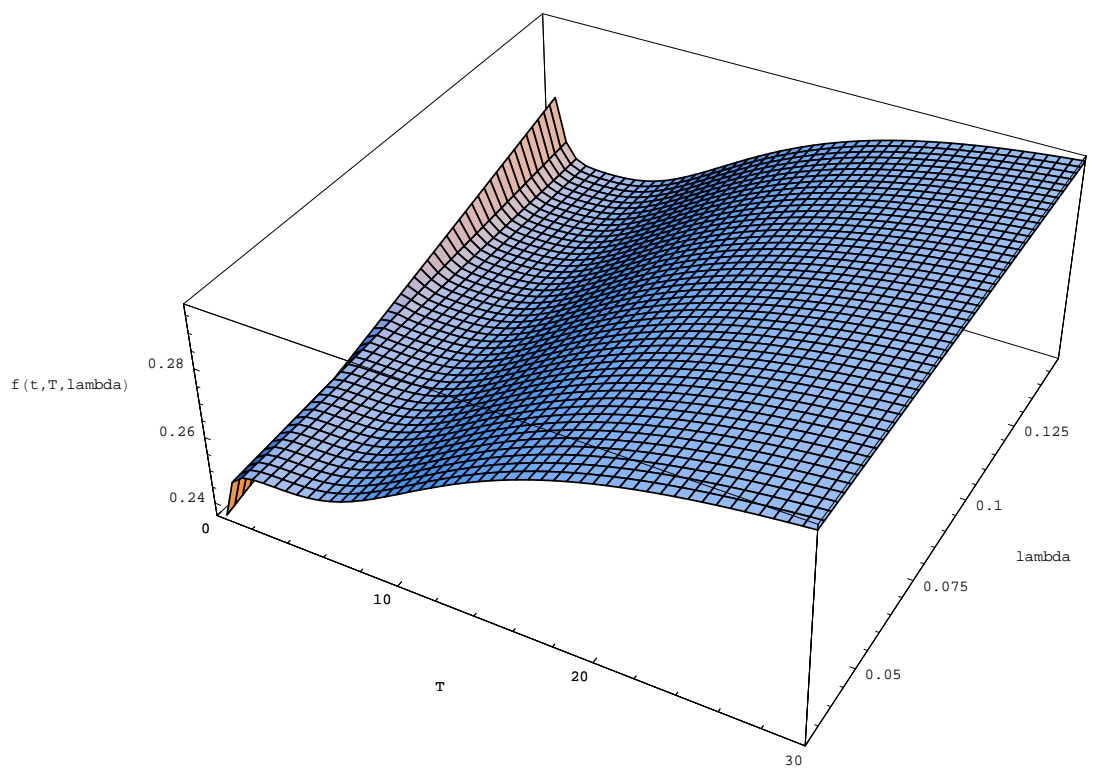

Figure 3.1: Defaultable forward rate curves.

such as Monte Carlo simulation. We estimate the initial zero-coupon defaultable bond price from (3.4) by the means of Monte Carlo simulations by calculating

$$
P^{d}(0, T)=\overline{\mathcal{Q}}_{0} E\left(\frac{\bar{S}_{0}^{\delta_{*}}}{\bar{S}_{T}^{\delta_{*}}} \exp \left\{-\int_{0}^{T}\left(r_{s}+\lambda_{s} m_{v}\right) d s\right\} \mid \mathcal{A}_{0}\right),
$$

where the discounted GOP $\bar{S}_{t}^{\delta_{*}}$ is driven by (3.8) and the jump intensity $\lambda_{t}$ follows the dynamics (3.14), with $E\left[d \tilde{W}_{t}, d W_{t}\right]=\rho d t$. Note that $\overline{\mathcal{Q}}_{0}=1$.

The challenge now lies on using an efficient simulation scheme. The standard discretisation schemes, for instance an Euler scheme, prove to be problematic. They allow the discounted GOP to take zero or even negative values, which results in meaningless prices. By using an exact simulation scheme, we can eliminate these problems. In Appendix D, we propose the exact simulation of two correlated squared Bessel processes as explained in Platen and Rendek (2009). Recall that the discounted GOP $\bar{S}_{t}^{\delta_{*}}$ is a time transformed squared Bessel process of dimension four with some deterministic transformed time. The CIR type dynamics (3.14) of the jump intensity can be expressed as the product of a deterministic factor and a squared Bessel process.

The proposed Monte Carlo simulation method, especially when $\rho \neq 0$, requires substantial computational effort to be reasonably accurate. To significantly reduce the statistical error of the simulation and consequently to minimise the computational effort, we discuss in the next subsection the effect of a variance reduction method. More efficient variance reduction methods are available, see for instance Heath and Platen (2002b), but these more complex methods are beyond 
the purpose of this work. Note that for $\rho=0$, we obtain the special case studied in Section 3.2, which provides an explicit pricing formula for defaultable bond prices. We use this special case as control variate in the evaluation of defaultable bonds for the more general dependent case. This explicit pricing formula can be of great value also in other variance reduction methods.

\subsection{Control Variate Method}

To demonstrate that variance reduction can be efficiently improved, we consider Monte Carlo simulation using a control variate method. By concurrent Monte Carlo simulations of both the independent and the dependent case, the simulated bond prices are derived which are denoted as $P_{\text {indep }}^{d}(0, T)$ and $P_{d e p}^{d}(0, T)$, respectively. We consider the outputs of $N \in \mathbb{N}$ Monte Carlo simulations. We denote as $\mathbb{I}_{1}, \mathbb{I}_{2}, \ldots, \mathbb{I}_{N}$ the outputs for the independent case and as $\mathbb{D}_{1}, \mathbb{D}_{2}, \ldots, \mathbb{D}_{N}$ the outputs for the more general dependent case. Then the bond price for the independent case is $P_{\text {indep }}^{d}(0, T)=E\left[\mathbb{I}_{i}\right]$ and the bond price for the dependent case is $P_{d e p}^{d}(0, T)=E\left[\mathbb{D}_{i}\right], i \in\{1,2, \ldots, N\}$. Denote as $(\mathbb{D}, \mathbb{I})$ a set of random variables with the same distribution as $\left(\mathbb{D}_{i}, \mathbb{I}_{i}\right)$.

Under the assumption of independence between the discounted GOP and the default-adjusted short rate, the closed form bond prices (3.15) are obtained which are denoted as $P_{\text {analytic }}^{d}(0, T)$ and represent the explicitly known expectation $E(\mathbb{I})$. Then for any constant $\alpha$, the sample mean

$$
P_{C V}^{d}(0, T)=\overline{\mathbb{D}}-\alpha[\overline{\mathbb{I}}-E(\mathbb{I})]=\frac{1}{N} \sum_{i=1}^{N}\left(\mathbb{D}_{i}-\alpha\left[\mathbb{I}_{i}-E(\mathbb{I})\right]\right),
$$

is an unbiased estimator for $P_{d e p}^{d}(0, T)$. The optimal coefficient $\alpha$, which minimizes the variance of the unbiased estimator $P_{C V}^{d}(0, T)$, is

$$
\alpha_{\text {min }}=\frac{\operatorname{cov}(\mathbb{I}, \mathbb{D})}{\operatorname{var}(\mathbb{I})}=\frac{\sum_{i=1}^{N}\left(\mathbb{I}_{i}-\overline{\mathbb{I}}\right)\left(\mathbb{D}_{i}-\overline{\mathbb{D}}\right)}{\sum_{i=1}^{N}\left(\mathbb{I}_{i}-\overline{\mathbb{I}}\right)^{2}},
$$

see for instance Clewlow and Carverhill (1992). Because the optimal value of $\alpha$ can only be approximated by estimating the quantities in (4.3), this will lead to an almost optimal control variate. For a study on minor effects on the efficiency of this kind of control variates see Lavenberg, Moeller, and Welch (1982).

Figure 4.2 shows the effect of the correlation coefficient $\rho$ on the value of $\alpha$. For our simulations we have used a value of $\alpha=1$, which seems reasonable, especially for $|\rho| \leq 0.5$.

Figure 4.3 compares the standard error of the simulated prices of $P_{d e p}^{d}(0, T)$ and $P_{C V}^{d}(0, T)$ for a range of different correlation coefficients. We assume zero recovery 
Alpha $(\mathrm{dt}=.01)$

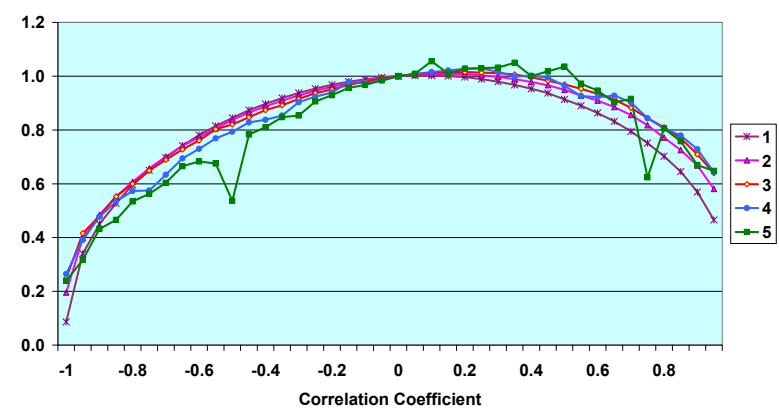

Alpha $(\mathrm{dt}=$.0025)

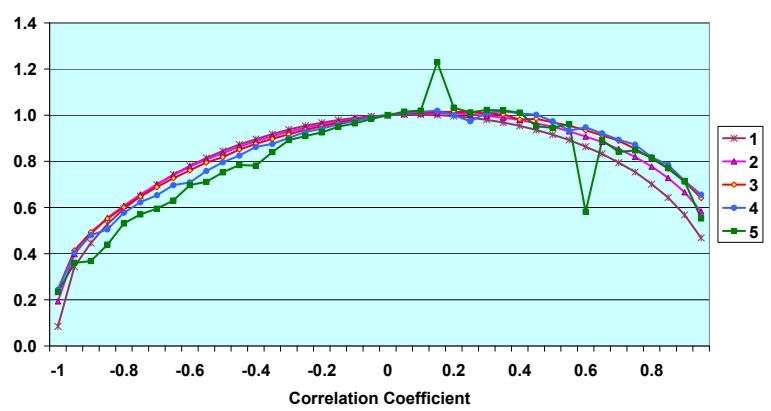

Figure 4.2: $\alpha$ as a function of $\rho$

and that the initial market price of risk is $\left|\theta_{0}\right|=\sqrt{\frac{\alpha_{0}}{\bar{S}_{0}}}=0.2$, the net growth rate is $\eta=0.05$ and $T=5$. Furthermore we assume that the short rate evolves as a deterministic function of time namely $d r_{t}=a\left(b-r_{t}\right) d t$, with the same parameter values as in Section 3.2.1. The parameter values of the default intensity (3.14) are $\bar{\lambda}=0.05, \sigma=0.15, \alpha=0.5, \kappa=0.45$ and $h=0.2$. Note that for these parameter values, the squared Bessel process modelling the default intensity is of dimension four. We run two set of simulations, with $N=1,000,000$ and $N=50,000,000$ respectively.

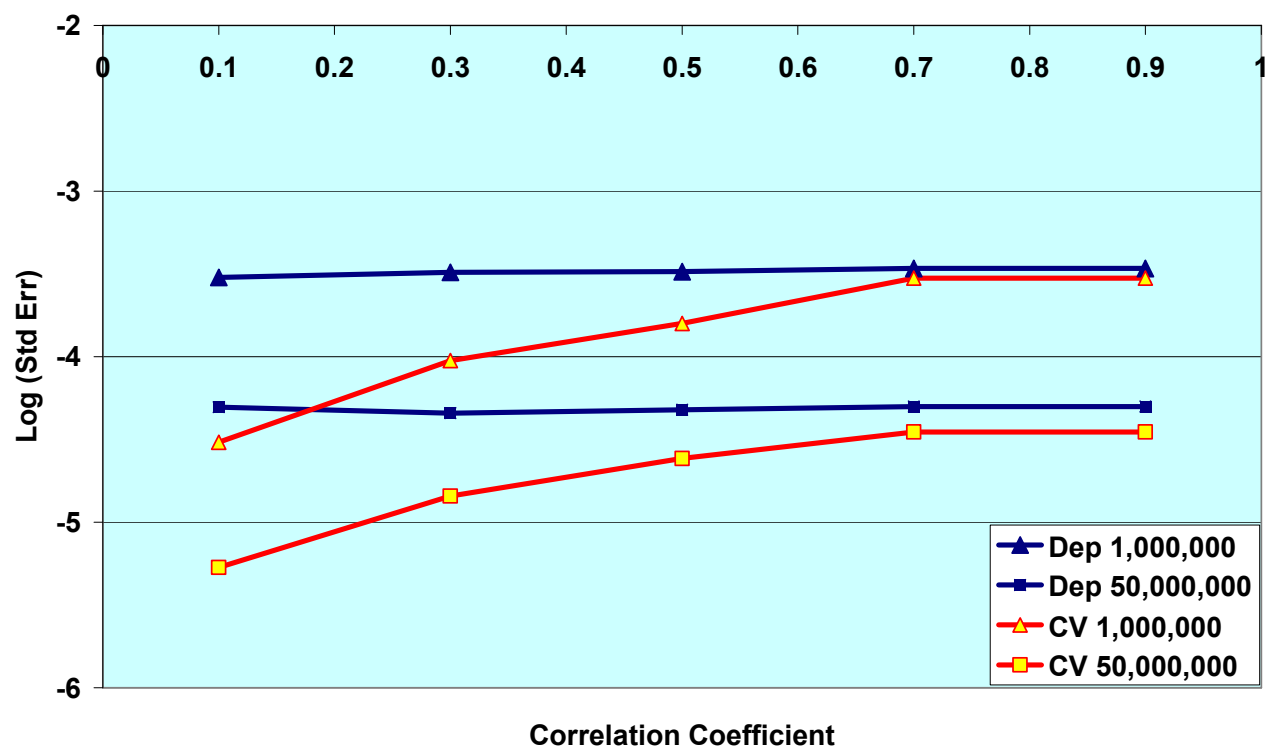

Figure 4.3: Log-log plot of the standard error for different correlation coefficients. Case "Dep": $P_{d e p}^{d}(0, T)$. Case "CV": $P_{C V}^{d}(0, T)$.

Figure 4.3 demonstrates that for low correlation between the discounted GOP and the default intensity, the standard error of the bond price estimates using the control variate method are approximately one tenth the magnitude of the values obtained by simulation of the dependent model without variance reduction. This 
reduction is uniform across the number of simulated paths and discretisation level. Thus, the same order of accuracy can be achieved by 100 times less the number of simulated paths. We confirm that by comparing the results for the different number of simulated paths, we observe that the results are consistent with the well-known fact that the standard errors decrease with $1 / \sqrt{N}$, at all reasonable discretization levels.

For $\rho=0.5$, the 5 -year bond prices estimated by the control variate method are of the order of at approximately one fifth with respect to the values obtained by the standard simulation scheme. However, for substantial correlation between the discounted GOP and the default intensity, for instance $\rho=0.9$, the control variates have no significant effect. Similar results are obtained for the defaultable bond prices when parameters have been selected such that the squared Bessel process modelling the default intensity is of dimension three or five.

Note that there exists a variety of powerful variance reduction techniques that one can employ to further improve the efficiency of the Monte Carlo simulation, see for instance Kloeden and Platen (1999) and Heath and Platen (2002b), however, this is beyond the scope of this paper.

\section{Conclusion}

This paper presents alternative defaultable term structure models formulated in a general setting in which the existence of an equivalent risk-neutral probability measure is not required. The real-world dynamics of the defaultable forward rates and the defaultable bond price are obtained, as well as finite dimensional Markovian defaultable term structure models under separable volatility specifications. Pricing in this general setting is accomplished by Monte Carlo simulation. Closed form solutions are derived under the assumption of independence between the discounted GOP and the default intensity, which turn out to be very usefull in this context. These can serve as control variates for variance reduction methods, which can significantly reduce the standard error of the Monte Carlo simulation for the more general models.

The proposed modelling approach holds great potential for more accurate credit risk assessment and measurement. It is a consistent approach under which pricing, hedging and credit risk measurement can be performed under a unique probability measure, the real-world probability measure. Therefore, we are able to perform a type of absolute pricing which has the significant advantage of estimating prices that reflect historical information and economic reasoning and not the common type of relative pricing. Real-world default probabilities can be evaluated by observed credit spreads, a feature that makes pricing and risk measurement more reliable and opens a line of further research. 


\section{Acknowledgement}

The authors would like to thank Carl Chiarella for his valuable comments. Furthermore, we would like to thank Troy Morgan for his significant contribution to the development of the numerical simulations.

\section{A Appendix: Proof of Proposition 2.2}

Let us define a "pseudo" zero-coupon bond $\tilde{P}(t, T)$, for $t \in[0, T]$ and $T \in[0, \bar{T}]$, $\bar{T} \in[0, \infty)$, which will be used in the following proofs. The pseudo zero-coupon bond $\tilde{P}(t, T)$ is the price of a defaultable zero-coupon bond maturing at $T$, which is identical to the defaultable bond $P(t, T)$, but has not defaulted up to time $t$. Then

$$
\tilde{P}(t, T)=\exp \left(-\int_{t}^{T} f^{d}(t, s) d s\right),
$$

and thus the value of the defaultable bond $P^{d}(t, T)(2.18)$ is given by

$$
P^{d}(t, T)=\tilde{P}(t, T) \overline{\mathcal{Q}}_{t} .
$$

Using the dynamics (2.8) and (2.12), an application of the Itô formula derives the dynamics of the pseudo zero-coupon bond $\tilde{P}(t, T)$ as

$$
\begin{aligned}
d \tilde{P}(t, T)=\tilde{P}(t-, T)[ & \left(r_{t}+\sum_{i=1}^{m} \tilde{\sigma}^{i}(t, T) \theta_{t}^{i}\right. \\
& \left.+\int_{0}^{1}[(1-v) \tilde{\beta}(v, t, T)-v](\psi(v, t)-1) \phi(d v, t)\right) d t \\
& \left.+\sum_{i=1}^{m} \tilde{\sigma}^{i}(t, T) d W_{t}^{i}+\int_{0}^{1} \tilde{\beta}(v, t, T) p(d v, d t)\right]
\end{aligned}
$$

for all $t \in[0, T]$, with

$$
\tilde{\sigma}^{i}(t, T)=\sigma^{i}(t, T)
$$

and

$$
\tilde{\beta}(v, t, T)=\frac{\beta(v, t, T)+v}{1-v}=\frac{\beta_{M}(v, t, T)}{1-v} .
$$

From the real-world dynamics (2.11) of the benchmarked defaultable bond price and the Itô formula, we obtain

$$
\begin{aligned}
& d \ln \left(\hat{P}^{d}(t, T)\right)=-\sum_{i=1}^{m}\left(\frac{1}{2}\left(\hat{\sigma}^{i}(t, T)\right)^{2} d t+\hat{\sigma}^{i}(t, T) d W_{t}^{i}\right) \\
& +\int_{0}^{1}(\hat{\beta}(v, t, T)+\ln (1-\hat{\beta}(v, t, T))) \phi(d v) d t+\int_{0}^{1} \ln (1-\hat{\beta}(v, t, T)) q(d v, d t) .
\end{aligned}
$$


Using definition (2.19) and (A.6), the defaultable forward rate dynamics under the real-world probability measure are

$$
\begin{aligned}
d f^{d}(t, T)= & \sum_{i=1}^{m} \frac{\partial}{\partial T} \hat{\sigma}^{i}(t, T)\left(\hat{\sigma}^{i}(t, T) d t+d W_{t}^{i}\right) \\
& +\int_{0}^{1} \frac{\frac{\partial}{\partial T} \hat{\beta}(v, t, T)}{1-\hat{\beta}(v, t, T)}(\hat{\beta}(v, t, T) \phi(d v) d t+q(d v, d t)),
\end{aligned}
$$

for $t \in[0, T]$. Next we express the dynamics of the forward rates (A.7) in terms of the diffusion coefficients (2.22) and the jump coefficient (2.23), where we have

$$
\begin{gathered}
\int_{t}^{T} \sigma_{d}^{i}(t, s) d s=\hat{\sigma}^{i}(t, T)-\hat{\sigma}^{i}(t, t) \\
\int_{t}^{T} \beta_{d}(v, t, s) d s=-\ln \left(\frac{1-\hat{\beta}(v, t, T)}{1-\hat{\beta}(v, t, t)}\right) .
\end{gathered}
$$

However, the "pseudo" bond volatilities (A.4) and (A.5) must satisfy the conditions (by definition (A.1), we have that $\tilde{P}(t, t)=1$, for $t \in[0, T]$ )

$$
\begin{aligned}
& 0=\tilde{\sigma}^{i}(t, t)=\sigma^{i}(t, t), \\
& 0=\tilde{\beta}(v, t, t)=\frac{\beta(v, t, t)+v}{1-v} .
\end{aligned}
$$

Using (2.13) and (2.14) we obtain that

$$
\begin{aligned}
& \sigma^{i}(t, t)=\theta_{t}^{i}-\hat{\sigma}^{i}(t, t)=0 \\
& \beta(v, t, t)=\frac{\psi(v, t)-\hat{\beta}(v, t, t)}{1-\psi(v, t)}=-v,
\end{aligned}
$$

therefore

$$
\begin{aligned}
& \hat{\sigma}^{i}(t, t)=\theta_{t}^{i}, \\
& \hat{\beta}(v, t, t)=\psi(v, t)+(1-\psi(v, t)) v .
\end{aligned}
$$

By (A.8) and (A.9), the benchmarked defaultable bond volatilities are linked to the defaultable forward rate volatilities by the relations

$$
\begin{aligned}
\hat{\sigma}^{i}(t, T) & =\int_{t}^{T} \sigma_{d}^{i}(t, s) d s+\theta_{t}^{i} \\
\hat{\beta}(v, t, T) & =1-\exp \left(-\int_{t}^{T} \beta_{d}(v, t, s) d s\right)(1-\psi(v, t))(1-v),
\end{aligned}
$$

which yields (2.20). 


\section{B Appendix: Proof of Equation (2.25)}

By equation (2.20), which we recall here

$$
d f^{d}(t, T)=\mu_{d}(t, T) d t+\sum_{i=1}^{m} \sigma_{d}^{i}(t, T) d W_{t}^{i}+\int_{0}^{1} \beta_{d}(v, t, T) p(d v, d t),
$$

and by using Proposition 2.2 of Björk, Kabanov, and Runggaldier (1997), we obtain the dynamics of the "pseudo" zero-coupon bond price $\bar{P}(t, T)$, defined in (A.1), as

$$
\begin{aligned}
& d \bar{P}(t, T)=\bar{P}\left(t^{-}, T\right)\left[\left(r_{t}^{d}-\int_{t}^{T} \mu_{d}(t, u) d u+\sum_{i=1}^{m} \frac{1}{2}\left(\int_{t}^{T} \sigma_{d}^{i}(t, u) d u\right)^{2}\right) d t\right. \\
& \left.-\sum_{i=1}^{m} \int_{t}^{T} \sigma_{d}^{i}(t, u) d u d W_{t}^{i}-\int_{0}^{1}\left(1-\exp \left\{-\int_{t}^{T} \beta_{d}(v, t, u) d u\right\}\right) p(d v, d t)\right] .
\end{aligned}
$$

Note that the defaultable spot rate $r_{t}^{d}$ is defined as $r_{t}^{d}=f^{d}(t, t)$, for all $t \in$ $[0, T]$. By comparison with the dynamics of the "pseudo" zero-coupon bond price obtained in (A.3), we derive the following relationships

$$
\begin{array}{r}
\bar{\sigma}^{i}(t, T)=-\int_{t}^{T} \sigma_{d}^{i}(t, u) d u, \\
\bar{\beta}(v, t, T)=-1+\exp \left\{-\int_{t}^{T} \beta_{d}(v, t, u) d u\right\},
\end{array}
$$

and the drift term $\bar{\mu}(t, T)$ is given by

$$
\bar{\mu}(t, T)=r_{t}^{d}-\int_{t}^{T} \mu_{d}(t, u) d u+\sum_{i=1}^{m} \frac{1}{2}\left(\int_{t}^{T} \sigma_{d}^{i}(t, u) d u\right)^{2},
$$

with (see (A.3))

$$
\bar{\mu}(t, T)=r_{t}+\sum_{i=1}^{m} \bar{\sigma}^{i}(t, T) \theta_{t}^{i}+\int_{0}^{1}[(1-v) \bar{\beta}(v, t, T)-v](\psi(v, t)-1) \phi(d v) .
$$

Using the relations (A.4), (2.13) and (A.10) we confirm that (B.3) holds since $\bar{\sigma}^{i}(t, T)=\sigma^{i}(t, T)=\theta_{t}^{i}-\hat{\sigma}^{i}(t, T)=\theta_{t}^{i}-\left(\int_{t}^{T} \sigma_{d}^{i}(t, s) d s+\theta_{t}^{i}\right)=-\int_{t}^{T} \sigma_{d}^{i}(t, s) d s$. 
Similarly, by using (A.5), (2.14) and (A.11)

$$
\begin{aligned}
\beta(v, t, T) & =\frac{\psi(v, t)-\hat{\beta}(v, t, T)}{1-\psi(v, t)} \\
& =\frac{\psi(v, t)-1+\exp \left(-\int_{t}^{T} \beta_{d}(v, t, s) d s\right)(1-\psi(v, t))(1-v)}{1-\psi(v, t)} \\
& =-1+\exp \left(-\int_{t}^{T} \beta_{d}(v, t, s) d s\right)(1-v) .
\end{aligned}
$$

Thus we confirm (B.4) since

$$
\bar{\beta}(v, t, T)=\frac{\beta(v, t, T)+v}{1-v}=-1+\exp \left\{-\int_{t}^{T} \beta_{d}(v, t, u) d u\right\} .
$$

From (B.3), (B.4), (B.5) and (B.6) we express the short rate spread as

$$
\begin{aligned}
r_{t}^{d}-r_{t}= & \int_{t}^{T} \mu_{d}(t, u) d u-\sum_{i=1}^{m} \frac{1}{2}\left(\int_{t}^{T} \sigma_{d}^{i}(t, u) d u\right)^{2} \\
& +\sum_{i=1}^{m} \bar{\sigma}^{i}(t, T) \theta_{t}^{i}+\int_{0}^{1}[(1-v) \bar{\beta}(v, t, T)-v](\psi(v, t)-1) \phi(d v) \\
= & \int_{t}^{T} \mu_{d}(t, u) d u-\sum_{i=1}^{m} \frac{1}{2}\left(\int_{t}^{T} \sigma_{d}^{i}(t, u) d u\right)^{2} \\
& -\sum_{i=1}^{m} \theta_{t}^{i} \int_{t}^{T} \sigma_{d}^{i}(t, s) d s+\int_{0}^{1}\left[(1-v) e^{-\int_{t}^{T} \beta_{d}(v, t, u) d u}-1\right](\psi(v, t)-1) \phi(d v) .
\end{aligned}
$$

By using the drift restriction (2.21)

$$
\begin{aligned}
\int_{t}^{T} \mu_{d}(t, u) d u= & \sum_{i=1}^{m} \int_{t}^{T} \sigma_{d}^{i}(t, u)\left(\int_{t}^{u} \sigma_{d}^{i}(t, s) d s+\theta_{t}^{i}\right) d u \\
& -\int_{t}^{T} \int_{0}^{1} \beta_{d}(v, t, u) \exp \left(-\int_{t}^{u} \beta_{d}(v, t, s) d s\right)(1-\psi(v, t))(1-v) \phi(d v) d u \\
= & \sum_{i=1}^{m} \int_{t}^{T} \sigma_{d}^{i}(t, u) \int_{t}^{u} \sigma_{d}^{i}(t, s) d s d u+\sum_{i=1}^{m} \theta_{t}^{i} \int_{t}^{T} \sigma_{d}^{i}(t, u) d u \\
& -\int_{t}^{T} \int_{0}^{1} \beta_{d}(v, t, u) \exp \left(-\int_{t}^{u} \beta_{d}(v, t, s) d s\right)(1-\psi(v, t))(1-v) \phi(d v) d u
\end{aligned}
$$

Note that

$$
\begin{aligned}
\int_{t}^{T} \sigma_{d}^{i}(t, u) \int_{t}^{u} \sigma_{d}^{i}(t, s) d s d u & =\frac{1}{2} \int_{t}^{T} \frac{\partial}{\partial u}\left(\int_{t}^{u} \sigma_{d}^{i}(t, s) d s\right)^{2} d s \\
& =\frac{1}{2}\left(\int_{t}^{T} \sigma_{d}^{i}(t, u) d u\right)^{2}
\end{aligned}
$$


Note also that

$$
\begin{aligned}
& \int_{t}^{T} \int_{0}^{1} \beta_{d}(v, t, u) \exp \left(-\int_{t}^{u} \beta_{d}(v, t, s) d s\right)(1-\psi(v, t))(1-v) \phi(d v) d u \\
& =\int_{0}^{1}(1-\psi(v, t))(1-v) \int_{t}^{T} \beta_{d}(v, t, u) \exp \left(-\int_{t}^{u} \beta_{d}(v, t, s) d s\right) d u \phi(d v) \\
& =\int_{0}^{1}(1-\psi(v, t))(1-v)\left[\exp \left(-\int_{t}^{T} \beta_{d}(v, t, s) d s\right)-1\right] \phi(d v) .
\end{aligned}
$$

Thus by (B.7), (B.8), (B.9) and (B.10) we obtain that

$$
\begin{aligned}
r_{t}^{d}-r_{t}= & \int_{0}^{1}(1-\psi(v, t))(1-v)\left[e^{-\int_{t}^{T} \beta_{d}(v, t, s) d s}-1\right] \phi(d v) \\
& +\int_{0}^{1}\left[(1-v) e^{-\int_{t}^{T} \beta_{d}(v, t, u) d u}-1\right](\psi(v, t)-1) \phi(d v) . \\
= & \int_{0}^{1}(1-\psi(v, t)) v \phi(d v) .
\end{aligned}
$$

\section{Appendix: Proof of Proposition 2.4}

Using the dynamics of the forward rate (2.20), and setting $t=T$, we can use the identity $r_{t}^{d}=f^{d}(t, t)$ to obtain the short rate dynamics

$$
r_{t}^{d}=f^{d}(0, t)+\int_{0}^{t} \mu_{d}(u, t) d u+\sum_{i=1}^{m} \int_{0}^{t} \sigma_{d}^{i}(u, t) d W_{u}^{i}+\int_{0}^{t} \int_{0}^{1} \beta_{d}(v, u, t) p(d v, d u),
$$

with $\mu_{d}$ as in (2.21). Thus

$$
\begin{aligned}
d r_{t}^{d}= & \left.\frac{\partial}{\partial T} f^{d}(t, T)\right|_{T=t} d t+\left.d f^{d}(t, T)\right|_{T=t} \\
= & {\left[\left.\frac{\partial}{\partial T} f^{d}(0, T)\right|_{T=t}+\right.} \\
+ & \left(\left.\int_{0}^{t} \frac{\partial}{\partial T} \mu_{d}(u, T)\right|_{T=t} d u+\left.\sum_{i=1}^{m} \int_{0}^{t} \frac{\partial}{\partial T} \sigma_{d}^{i}(u, T)\right|_{T=t} d W_{u}^{i}\right. \\
& \left.\left.\quad+\left.\int_{0}^{t} \int_{0}^{1} \frac{\partial}{\partial T} \beta_{d}(v, u, T)\right|_{T=t} p(d v, d u)\right)\right] d t \\
+ & \mu_{d}(t, t) d t+\sum_{i=1}^{m} \sigma_{d}^{i}(t, t) d W_{t}^{i}+\int_{0}^{1} \beta_{d}(v, t, t) p(d v, d t) .
\end{aligned}
$$

Using the volatility specifications of Assumption 2.3, we obtain

$$
\left.\frac{\partial \sigma_{d}^{i}(u, T)}{\partial T}\right|_{T=t}=-k_{\sigma}^{i}(t) \sigma_{d}^{i}(u, t)
$$


and

$$
\left.\frac{\partial \beta_{d}(v, u, T)}{\partial T}\right|_{T=t}=-k_{\beta}(t) \beta_{d}(v, u, t)
$$

By introducing

$$
V_{\sigma}^{i}(u, T)=\sigma_{d}^{i}(u, T) \int_{u}^{T} \sigma_{d}^{i}(u, s) d s
$$

and

$$
V_{\beta}(v, u, T)=(1-v) \beta_{d}(v, u, T) \exp \left(-\int_{u}^{T} \beta_{d}(v, u, s) d s\right),
$$

we have by (2.21) that

$$
\mu_{d}(u, T)=\sum_{i=1}^{m} V_{\sigma}^{i}(u, T)+\sum_{i=1}^{m} \sigma_{d}^{i}(u, T) \theta_{u}^{i}-\int_{0}^{1} V_{\beta}(v, u, T)(1-\psi(v, u)) \phi(d v, t) .
$$

Also note that by $(2.27)$, for $i \in\{1,2, \ldots, m\}$,

$$
\left.\frac{\partial V_{\sigma}^{i}(u, T)}{\partial T}\right|_{T=t}=-k_{\sigma}^{i}(t) V_{\sigma}^{i}(u, t)+\left(\sigma_{d}^{i}(u, t)\right)^{2},
$$

and by (2.28)

$$
\left.\frac{\partial V_{\beta}(v, u, T)}{\partial T}\right|_{T=t}=-k_{\beta}(t) V_{\beta}(v, u, t)-(1-v)\left(\beta_{d}(v, u, t)\right)^{2} e^{-\int_{u}^{t} \beta_{d}(v, u, s) d s} .
$$

Using the above results, the dynamics of the spot rate (C.2) are expanded to

$$
\begin{aligned}
d r_{t}^{d} & =\left\{\left.\frac{\partial}{\partial T} f^{d}(0, T)\right|_{T=t}+\int_{0}^{t} \sum_{i=1}^{m}\left[-k_{\sigma}^{i}(t) V_{\sigma}^{i}(u, t)+\left(\sigma_{d}^{i}(u, t)\right)^{2}-k_{\sigma}^{i}(t) \sigma_{F}^{i}(u, t) \theta_{u}^{i}\right] d u\right. \\
& +\int_{0}^{t} \int_{0}^{1}\left[\left\{k_{\beta}(t) V_{\beta}(v, u, t)+(1-v)\left(\beta_{d}(v, u, t)\right)^{2} e^{-\int_{u}^{t} \beta_{d}(v, u, s) d s}\right\}(1-\psi(v, u)) \phi(d v, t)\right] d u \\
& \left.-\sum_{i=1}^{m} k_{\sigma}^{i}(t) \int_{0}^{t} \sigma_{d}^{i}(u, t) d W_{u}^{i}-k_{\beta}(t) \int_{0}^{t} \int_{0}^{1} \beta_{d}(v, u, t) p(d v, d u)\right\} d t \\
& +\left(\sum_{i=1}^{m} \sigma_{d}^{i}(t, t) \theta_{t}^{i}-\int_{0}^{1} \beta_{d}(v, t, t)(1-v)(1-\psi(v, t)) \phi(d v, t)\right) d t \\
& +\sum_{i=1}^{m} \sigma_{d}^{i}(t, t) d W_{t}^{i}+\int_{0}^{1} \beta_{d}(v, t, t) p(d v, d t) .
\end{aligned}
$$

By using the definitions (2.30), (2.31), (2.32) and (2.33), (C.10) reduces to

$$
\begin{aligned}
d r_{t}^{d}=\{ & \left.\frac{\partial}{\partial T} f^{d}(0, T)\right|_{T=t}+\mathcal{E}_{\beta}(t)+\sum_{i=1}^{m} \mathcal{E}_{\sigma}^{i}(t)-\sum_{i=1}^{m} k_{\sigma}^{i}(t) \mathcal{D}_{\sigma}^{i}(t)-k_{\beta}(t) \mathcal{D}_{\beta}(t) \\
& \left.+\sum_{i=1}^{m} \bar{\sigma}_{d}^{i}\left(t, \mathrm{~F}_{t}\right) \theta_{t}^{i}\right\} d t+\sum_{i=1}^{m} \bar{\sigma}_{d}^{i}\left(t, \mathrm{~F}_{t}\right) d W_{t}^{i}+\int_{0}^{1} \bar{\beta}_{d}(v, t) p(d v, d t)
\end{aligned}
$$


Further from definitions (2.31) and (2.32), (C.1) yields

$$
\mathcal{D}_{\sigma}^{1}(t)=r_{t}^{d}-f^{d}(0, t)-\sum_{i=2}^{m} \mathcal{D}_{\sigma}^{i}(t)-\mathcal{D}_{\beta}(t),
$$

which reduces (C.11) to (2.29).

\section{Simulation of Correlated Squared Bessel Pro- cesses}

The objective is to simulate the discounted GOP and the default adjusted short rate by allowing correlated dynamics. The dynamics (3.8) drive the discounted GOP, recall

$$
d \bar{S}_{t}^{\delta_{*}}=\bar{S}_{t-}^{\delta_{*}} \theta_{t}\left(\theta_{t} d t+d W_{t}\right)
$$

with the net market trend defined by

$$
\alpha_{t}=\bar{S}_{t}^{\delta_{*}}\left|\theta_{t}\right|^{2}
$$

Note that the total market price of risk can be expressed as

$$
\left|\theta_{t}\right|=\sqrt{\frac{\alpha_{t}}{\bar{S}_{t}^{\delta_{*}}}}
$$

and the dynamics (D.1) are

$$
d \bar{S}_{t}^{\delta_{*}}=\alpha_{t} d t+\sqrt{\alpha_{t} \bar{S}_{t}^{\delta_{*}}} d W_{t}
$$

By introducing the transformed time $\varphi_{t}^{1}$ satisfying

$$
d \varphi_{t}^{1}=\frac{1}{4} \int_{0}^{t} \alpha_{s} d s
$$

then the process $X_{\varphi_{t}^{1}}=\bar{S}_{t}^{\delta_{*}}$ has dynamics

$$
d X_{\varphi_{t}^{1}}=4 d \varphi_{t}^{1}+2 \sqrt{X_{\varphi_{t}^{1}}} d W\left(\varphi_{t}^{1}\right)
$$

where $d W\left(\varphi_{t}^{1}\right)=\sqrt{\frac{\alpha_{t}}{4}} d W_{t}$. This is the SDE of a square Bessel process of dimension 4. Under the MMM proposed by Platen (2001) and Platen (2002), a deterministic time transformation is assumed with the net market trend $\alpha_{t}$, $t \in[0, T]$ having the form

$$
\alpha_{t}=\alpha_{0} \exp \{\eta t\}
$$


where $\eta>0$ is the constant net growth rate and $\alpha_{0}>0$ is an initial scaling parameter. Then the transformed time is reduced to

$$
\varphi_{t}^{1}=\varphi_{0}^{1}+\frac{\alpha_{0}}{4 \eta}\left(e^{\eta t}-1\right) .
$$

$\left(\varphi_{0}^{1}=0\right)$ Next consider the normalised GOP

$$
Y_{t}=\frac{\bar{S}_{t}^{\delta_{*}}}{\alpha_{t}}
$$

for $t \in[0, \infty)$. Then by the Itô formula we obtain

$$
d Y_{t}=\left(1-\eta Y_{t}\right) d t+\sqrt{Y_{t}} d W_{t}
$$

for $t \in[0, \infty)$ and $Y_{0}=\frac{\bar{S}_{0}^{\delta_{*}}}{\alpha_{0}}$. Therefore from (D.9), the discounted GOP can be expressed as the product of the exponential function (D.7) and the square root process (D.10).

The stochastic jump intensity $\lambda_{t}$ follows the dynamics (3.14) recall

$$
d \lambda_{t}=\kappa\left(\bar{\lambda}-\lambda_{t}\right) d t+\sigma \sqrt{\lambda_{t}} d \tilde{W}_{t}+d J_{t},
$$

where $\kappa, \bar{\lambda}, \sigma$ are positive constants, with $2 \kappa \bar{\lambda}>\sigma^{2}$. Between the jump times, we need to simulate the continuous part of these dynamics, namely

$$
d \lambda_{t}=\kappa\left(\bar{\lambda}-\lambda_{t}\right) d t+\sigma \sqrt{\lambda_{t}} d \tilde{W}_{t}
$$

Let us introduce the exponential function $s_{t}$

$$
s_{t}=s_{0} \exp (-\kappa t)
$$

and the transformed time

$$
\varphi_{t}^{2}=\varphi_{0}^{2}+\frac{1}{4} \int_{0}^{t} \frac{\sigma^{2}}{s_{u}} d u=\varphi_{0}^{2}+\frac{\sigma^{2}}{4 \kappa s_{0}}(\exp (\kappa t)-1) .
$$

Then the square root process (D.12) can be expressed as the product of the exponential function (D.13) $s_{t}$ and the squared Bessel process $\tilde{X}$ of dimension $\delta=\frac{4 \kappa \bar{\lambda}}{\sigma^{2}}>0$ by the transformation

$$
\lambda_{t}=s_{t} \tilde{X}_{\varphi_{t}^{2}}
$$

for $t \in[0, \infty)$. Note that

$$
d \tilde{X}_{\varphi_{t}^{2}}=\delta d \varphi_{t}^{2}+2 \sqrt{\tilde{X}_{\varphi_{t}^{2}}} d \tilde{W}\left(\varphi_{t}^{2}\right)
$$

where $d \tilde{W}\left(\varphi_{t}^{2}\right)=\sqrt{\frac{\sigma^{2}}{4 s_{t}}} d \tilde{W}_{t}$. 
We illustrate next the simulation of these two correlated squared Bessel processes. Recall that $E\left[d \tilde{W}_{t}, d W_{t}\right]=\rho d t$. The discounted GOP is a squared Bessel process of dimension 4 and we assume that the default adjusted short rate is a squared Bessel process of dimension $\delta \in\{1,2,3, \ldots\}$. In general the dimension $\delta$ can be any nonnegative real number, however we restrict it to an integer to be able to simulate two correlated squared Bessel processes as explained in Renata and Platen. Motivated by the $2 \times 2$ matrix time changed Wishart process where the diagonal elements of the process formulate correlated time changed squared Bessel processes of the same dimension, we consider a more general setting. We consider the two time changed matrix Wiener processes $\mathbf{W}_{\varphi_{t}^{1}}=\left[W_{\varphi_{t}^{1}}^{i j}\right]_{i, j=1}^{4,2}$ with $\varphi_{t}^{1}$ defined by (D.8) and $\tilde{\mathbf{W}}_{\varphi_{t}^{2}}=\left[\tilde{W}_{\varphi_{t}^{2}}^{i, j}\right]_{i, j=1}^{\delta, 2}$ with $\varphi_{t}^{2}$ defined by (D.14). Renata and Platen have illustrated that correlated time changed squared Bessel processes can be constructed as follows:

$$
\begin{aligned}
\bar{S}_{t}^{\delta_{*}} & =X_{\varphi_{t}^{1}} \\
& = \begin{cases}\sum_{i=1}^{\delta}\left(w^{i, 1}+\varrho W_{\varphi_{t}^{1}}^{i, 1}+\sqrt{1-\varrho^{2}} W_{\varphi_{t}^{1}}^{i, 2}\right)^{2}+\sum_{i=\delta+1}^{4}\left(w^{i, 1}+W_{\varphi_{t}^{1}}^{i, 1}\right), & \delta \leq 4 ; \\
\sum_{i=1}^{4}\left(w^{i, 1}+\varrho W_{\varphi_{t}^{1}}^{i, 1}+\sqrt{1-\varrho^{2}} W_{\varphi_{t}^{i 1}}^{i, 2}\right. & \delta>4 .\end{cases}
\end{aligned}
$$

and

$$
\frac{\lambda_{t}}{s_{t}}=\tilde{X}_{\varphi_{t}^{2}}=\sum_{i=1}^{\delta}\left(w^{i, 2}+\tilde{W}_{\varphi_{t}^{2}}^{i, 1}\right)^{2},
$$

where $X_{0}=\sum_{i=1}^{4}\left(w^{i, 1}\right)^{2}$ and $\tilde{X}_{0}=\sum_{i=1}^{\delta}\left(w^{i, 2}\right)^{2}$. This setup depends on the dimension $\delta$ of the squared Bessel process modelling the default adjusted short rate.

Let consider the time $t=\left\{t_{0}, t_{1}, \ldots, t_{N}=T\right\}$, then the time $\varphi_{t}$ is defined as $\varphi_{t}=\left\{\varphi_{t_{0}}, \varphi_{t_{1}}, \ldots, \varphi_{T}\right\}$, where $\varphi_{t}$ is determined here by (D.8) or (D.14). The $\varphi-$ time increment is $\Delta_{\varphi}=\varphi_{t_{i}}-\varphi_{t_{i-1}}$, therefore

$$
W_{\varphi_{t}}^{i}=W_{\varphi_{t}}^{i-1}+\sqrt{\Delta_{\varphi}} x, \text { with } x \sim N(0,1) .
$$

For instance if the $t$-time increment is $\Delta=\frac{T}{N}$, then the $t=\{0, \Delta, 2 \Delta, \ldots, N \Delta=$ $T\}$, and the $\varphi_{t}=\left\{\varphi_{0}, \varphi_{\Delta}, \varphi_{2 \Delta}, \ldots, \varphi_{T}\right\}$, therefore $\Delta_{\varphi}=\varphi_{i \Delta}-\varphi_{(i-1) \Delta}$. 


\section{References}

Albanese, C. and O. X. Chen (2005). Discrete credit barrier models. Quantitative Finance 5(3), 247-256.

Björk, T., Y. Kabanov, and W. Runggaldier (1997). Bond market structure in the presence of market point processes. Mathematical Finance 7, 211-239.

Broadie, M. and O. Kaya (2006). Exact simulation of stochastic volatility and other affine jump diffusion processes. Operations Research 54(2), 217-231.

Bruti-Liberati, N., C. Nikitopoulos-Sklibosios, and E. Platen (2007). Pricing under the real-world probability measure for jump-diffusion term structure models. Technical report, University of Technology, Sydney. QFRC Research Paper 198, Quantitative Finance to appear.

Chiarella, C., E. Schlögl, and C. S. Nikitopoulos (2007). A Markovian Defaultable Term Structure Model with State Dependent Volatilities. International Journal of Theoretical and Applied Finance 10(1), 155-202.

Christensen, M. M. and E. Platen (2005). A general benchmark model for stochastic jump sizes. Stochastic Analysis and Applications 23(5), 10171044 .

Clewlow, L. and A. Carverhill (1992). Efficient Monte Carlo valuation and hedging of contingent claims. Technical report, Financial Options Research Centre, University of Warwick. 92/30.

Duffie, D. (2005). Credit risk modelling with affine processes. Journal of Banking and Finance 29, 2751-2802.

Duffie, D. and K. Singleton (1999). Modeling term structures of defaultable bonds. Review of Financial Studies 12, 687-720.

Elliott, R. J., M. Jeanblanc, and M. Yor (2000). On models on default risk. Mathematical Finance 10(2), 179-196.

Filipović, D. (2001). A general characterization of one factor affine term structure models. Finance and Stochastics 5, 389-412.

Heath, D., R. Jarrow, and A. Morton (1992). Bond pricing and the term structure of interest rates: A new methodology for contingent claim valuation. Econometrica 60(1), 77-105.

Heath, D. and E. Platen (2002a). Perfect hedging of index derivatives under a minimal market model. International Journal of Theoretical and Applied Finance 5(7), 757-774.

Heath, D. and E. Platen (2002b). A variance reduction technique based on integral representations. Quantitative Finance 2(5), 362-369.

Karatzas, I. and S. E. Shreve (1998). Methods of Mathematical Finance, Volume 39. Springer. 
Kelly, J. R. (1956). A new interpretation of information rate. Bell System Technical Journal 35, 917-926.

Kloeden, P. E. and E. Platen (1999). Numerical Solution of Stochastic Differential Equations, Volume 23. Springer. Third corrected printing.

Lavenberg, S., T. Moeller, and P. Welch (1982). Statistical results on control variables with application to queueing network simulation. Operations Research 30, 182-202.

Long, J. B. (1990). The numeraire portfolio. Journal of Financial Economics 26, 29-69.

Matacz, A. and J. P. Bouchaud (2000). An empirical investigation of the forward interest rate term structure. International Journal of Theoretical and Applied Finance 3(4), 703-729.

Platen, E. (2001). A minimal financial market model. In Trends in Mathematics, pp. 293-301. Birkhäuser.

Platen, E. (2002). Arbitrage in continuous complete markets. Advances in Applied Probability 34(3), 540-558.

Platen, E. (2005). An alternative interest rate term structure model. International Journal of Theoretical and Applied Finance 8(6), 717-735.

Platen, E. and D. Heath (2006). A Benchmark Approach to Quantitative Finance. Springer.

Platen, E. and R. Rendek (2009). Exact scenario simulation for selected multidimensional stochastic processes. UTS (working paper).

Schönbucher, P. J. (1998). Term structure modelling of defaultable bonds. Review of Derivatives Research 2, 161-192.

Schönbucher, P. J. (2003). Credit Derivatives Pricing Models. Wiley, Chichester. 